\title{
PERIODIC POINTS OF POSITIVELY EXPANSIVE MAPS
}

\author{
KAZU-HIRO SAKAI
}

\begin{abstract}
Stability of fixed points of a uniformly convergent sequence of open $\varepsilon$-locally expansive maps of a compact connected locally connected metric space is studied by $\mathrm{Hu}$ and Rosen. Under the same assumption we prove a stability result stronger than theirs.
\end{abstract}

$\mathrm{Hu}$ and Rosen proved [4] a stability theorem for fixed points of a uniformly convergent sequence of open $\varepsilon$-locally expansive maps of a compact connected locally connected metric space. We prove a stronger stability theorem by using the pseudo-orbit tracing property.

Let $(X, d)$ be a compact metric space, and let $f: X \rightarrow X$ be a continuous map. We assume that $f$ is onto. We say that $f$ is positively expansive if there exists a number $c>0$ such that $x \neq y$ implies the existence of a positive integer $n$ such that $d\left(f^{n}(x), f^{n}(y)\right)>c$; $c$ is called a positively expansive constant for $f$. We say that $f$ is $\varepsilon$-locally expansive if there is an $\varepsilon>0$ such that $0<d(x, y)<\varepsilon$ implies $d(f(x), f(y))>d(x, y)$. We say that $f$ is an $\varepsilon$-local expansion with skewness $\lambda$ if there exist $\varepsilon>0$ and $\lambda>1$ such that $0<d(x, y)<\varepsilon$ implies $d(f(x), f(y))>$ $\lambda d(x, y)$. In [5] Reddy proved that if $f$ is $\varepsilon$-locally expansive, then $f$ is positively expansive with constant $\varepsilon / 2$, and there is a compatible metric $\rho$ for $X$ such that $f$ is an $\varepsilon_{1}$-local expansion with skewness $\lambda$ under $\rho$ for some constants $\varepsilon_{1}>0$ and $\lambda>1$. Rosenholtz showed in [7] that if $f$ is an open $\varepsilon$-local expansion and if $X$ is connected, then $f$ has a fixed point. Given $\delta>0$, a sequence $\left\{x_{j}\right\}_{j=0}^{n}(0 \leqslant n \leqslant \infty)$ is called a $\delta$-pseudo-orbit ( $\delta$-p.o.) of $f$ if $d\left(f\left(x_{j}\right), x_{j+1}\right)<\delta$ for $0 \leqslant j \leqslant n-1$. Given $\eta>0$, a sequence $\left\{x_{j}\right\}_{j=0}^{n}$ is said to be $\eta$-traced by a point $y$ in $X$ if $d\left(f^{j}(y), x_{j}\right)<\eta$ for $0 \leqslant j \leqslant n$. We say that $f$ has the pseudo-orbit tracing property (P.O.T.P.) if for each $\eta>0$ there is $\delta>0$ such that every $\delta$-p.o. of $f$ can be $\eta$-traced by some point in $X$. We remark that this property is independent of the metric for $X$. It is well known that if $f$ is an open $\varepsilon$-local expansion, then $f$ has the P.O.T.P. (cf. Proposition 3.6 of [2]).

The following is proved.

THEOREM. Let $(X, d)$ be a compact connected locally connected metric space, and let $f_{i}: X \rightarrow X$ be open $\varepsilon$-locally expansive maps for $i=0,1,2, \ldots$ such that the sequence

Received by the editors October 17, 1983 and, in revised form, July 6, 1984.

1980 Mathematics Subject Classification. Primary 54H25, 54E40, 54A20.

Key words and phrases. Periodic points, positively expansive maps, compact connected metric spaces, $\varepsilon$-locally expansive maps, $\varepsilon$-local expansions, pseudo-orbit tracing property (P.O.T.P.) 
$\left\{f_{i}\right\}_{i=1}^{\infty}$ converges uniformly to $f_{0}$. Then for any $\eta>0$ there is a positive integer $N$ such that, for each $n \geqslant 1$ and each fixed point $a_{0}$ of $f_{0}^{n}$, there exists a sequence of fixed points $a_{i}$ of $f_{i}^{n}$ with $d\left(a_{i}, a_{0}\right)<\eta$ for each $i \geqslant N$.

First of all we prepare lemmas that we shall need.

Lemma 1. Let $(X, \rho)$ be a compact metric space, and let $f: X \rightarrow X$ be an open $\varepsilon_{1}$-local expansion with skewness $\lambda$. Then there is a positive number $\delta_{0}<\varepsilon_{1} / 2$ such that $x, y \in X$ and $\rho(f(x), y)<\delta_{0}$ implies $B_{\delta_{0} / \lambda}(x) \cap f^{-1}(y) \neq \varnothing$, where $B_{\alpha}(x)=\{z \in$ $X: \rho(x, z)<\alpha\}$.

Proof. See Lemma 1 of [3].

LEMMA 2. Let $(X, \rho)$ be a compact connected metric space, and let $f: X \rightarrow X$ be an open $\varepsilon_{1}$-local expansion with skewness $\lambda$. Then $\operatorname{per}(f)$, the set of all periodic points of $f$, is dense in $X$.

Proof. Let $\delta_{0}$ be as in Lemma 1. It is enough to see that for each $\nu>0\left(\nu<\delta_{0} / 2\right)$ there is a positive integer $r$ such that $f^{r}$ has a fixed point in $B_{\nu}(x)$. For each number $\nu>0$, choose $\delta>0$ as in the definition of the P.O.T.P. Let $\mathscr{U}=\left\{U_{1}, U_{2}, \ldots, U_{n}\right\}$ be a finite open cover of $X$ so that the diameter of each $U_{i} \in \mathscr{U}$ is less than $\delta_{0}$. Then we can choose a positive integer $r$ such that $n \delta_{0} / \lambda^{r-1}<\delta$. Take and fix $x_{0} \in X$. Since $X$ is connected, there is a finite sequence $\left\{y_{0}=x_{0}, y_{1}, \ldots, y_{k}, y_{k+1}=f^{r}\left(x_{0}\right)\right\}$ such that $y_{j}, y_{j+1} \in U_{i_{j}} \in \mathscr{U}$ for $0 \leqslant j \leqslant k(\leqslant n)$. By Lemma 1 there is $y_{k}^{1}$ in $X$ with the property that $f\left(y_{k}^{1}\right)=y_{k}$ and $\rho\left(y_{k}^{1}, f^{r-1}\left(x_{0}\right)\right)<\delta_{0} / \lambda$ (since $\rho\left(y_{k}, f\left(f^{r-1}\left(x_{0}\right)\right)\right)<$ $\left.\delta_{0}\right)$. Similarly, since $\rho\left(y_{k-1}, f\left(y_{k}^{1}\right)\right)<\delta_{0}$, there is $y_{k-1}^{1}$ with the property that $f\left(y_{k-1}^{1}\right)=y_{k-1}$ and $\rho\left(y_{k-1}^{1}, y_{k}^{1}\right)<\delta_{0} / \lambda$. Continuing in this fashion, we can construct a finite sequence $\left\{y_{0}^{1}=x_{1}, y_{1}^{1}, \ldots, y_{k}^{1}, y_{k+1}^{1}=f^{r-1}\left(x_{0}\right)\right\}$. Next, since $\rho\left(y_{k}^{1}, f^{r-1}\left(x_{0}\right)\right)<\delta_{0}$, by Lemma 1 there is $y_{k}^{2}$ in $X$ with the property that $f\left(y_{k}^{2}\right)=y_{k}^{1}$ and $\rho\left(y_{k}^{2}, f^{r-2}\left(x_{0}\right)\right)<\delta_{0} / \lambda^{2}$. Similarly, since $\rho\left(y_{k-1}^{1}, f\left(y_{k}^{2}\right)\right)<\delta_{0} / \lambda$, there is $y_{k-1}^{2}$ in $X$ with the property that $f\left(y_{k-1}^{2}\right)=y_{k-1}^{1}$ and $\rho\left(y_{k-1}^{2}, y_{k}^{2}\right)<\delta_{0} / \lambda^{2}$. In this manner we can find a finite sequence $\left\{y_{0}^{2}=x_{2}, y_{1}^{2}, \ldots, y_{k}^{2}, y_{k-1}^{2}=f^{r-2}\left(x_{0}\right)\right\}$. Inductively, for $1 \leqslant l \leqslant r-1$, we can construct a finite sequence $\left\{y_{0}^{l}=x_{l}, y_{1}^{l}, \ldots, y_{k}^{l}, y_{k+1}^{l}=\right.$ $\left.f^{r-1}\left(x_{0}\right)\right\}$. Then

$$
\rho\left(x_{r-1}, f\left(x_{0}\right)\right) \leqslant \rho\left(y_{0}^{r-1}, y_{1}^{r-1}\right)+\cdots+\rho\left(y_{k}^{r-1}, f\left(x_{0}\right)\right) \leqslant n \delta_{0} / \lambda^{r-1}<\delta .
$$

Hence, $\left\{x_{0}, x_{r-1}, x_{r-2}, \ldots, x_{1}, x_{0}, x_{r-1}, \ldots\right\}$ is a $\delta$-p.o. of $f$. Since $f$ has the P.O.T.P. and $f$ is positively expansive with constant $\delta_{0}$, there is a tracing point $p$ in $B_{\nu}\left(x_{0}\right)$ such that $f^{r}(p)=p$ (cf. [1]).

Lemma 3. Let $(X, d)$ be a compact connected metric space, and let $f: X \rightarrow X$ be open and $\varepsilon$-locally expansive. If $E$ is a closed nonempty subset of $X$ with $f^{-1}(E) \subset E$, then $E=X$.

Proof. There exist a compatible metric $\rho$ for $X$ and numbers $\varepsilon_{1}>0$ and $\lambda>1$ such that $f$ is an open $\varepsilon_{1}$-local expansion with skewness $\lambda$ under $\rho$. To arrive at the conclusion, it is enough to prove that $\operatorname{per}(f) \subset E$. Let $p \in \operatorname{per}(f)$ with $f^{r}(p)=p$. Since $X$ is compact, there are $\varepsilon^{\prime}>0$ and $\lambda^{\prime}>1$ such that $f^{r}$ is an open $\varepsilon^{\prime}$-local 
expansion with skewness $\lambda^{\prime}$ under $\rho$. Hence, we can find $\delta_{0}^{\prime}>0$ satisfying Lemma 1 for $f^{r}$. Let $\mathscr{U}^{\prime}=\left\{U_{1}^{\prime}, U_{2}^{\prime}, \ldots, U_{n^{\prime}}^{\prime}\right\}$ be a finite open cover of $X$ so that the diameter of each $U_{i_{j}}^{\prime} \in \mathscr{U}^{\prime}$ is less than $\delta_{0}^{\prime}$ under $\rho$. Take $y_{0} \in E$. Since $X$ is connected, there is a finite sequence $\left\{z_{0}=y_{0}, z_{1}, \ldots, z_{k^{\prime}}, z_{k^{\prime}+1}=f^{r}(p)=p\right\}$ such that $z_{j}, z_{j+1} \in U_{i_{j}}^{\prime}$ for $0 \leqslant j \leqslant k^{\prime}\left(\leqslant n^{\prime}\right)$. By the argument used in the proof of Lemma 2, for $m>0$ we can construct a finite sequence $\left\{z_{0}^{m}=y_{m}, z_{1}^{m}, \ldots, z_{k^{\prime}}^{m}, z_{k^{\prime}+1}^{m}=f^{r}(p)=p\right\}$ with the property that $y_{m} \in f^{-r m}\left(y_{0}\right) \subset E$ and $\rho\left(y_{m}, z_{1}^{m}\right)+\cdots+\rho\left(z_{k^{\prime}}^{m}, p\right) \leqslant n^{\prime} \delta_{0}^{\prime} / \lambda^{\prime m}$. Hence, $y_{m} \rightarrow p(m \rightarrow \infty)$, so $\operatorname{per}(f) \subset E$.

The following lemma is essentially due to $\mathrm{Hu}$ and Rosen [4].

LEMMA 4. Let $(X, d)$ be a compact connected locally connected metric space, and let $f_{i}: X \rightarrow X$ be open E-locally expansive maps for $i=0,1,2, \ldots$ such that the sequence $\left\{f_{i}\right\}_{i=1}^{\infty}$ converges uniformly to $f_{0}$. Then there is an integer $N_{1}>0$ such that $i \geqslant N_{1}$ implies card $\left(f_{i}^{-1}(x)\right)=\operatorname{card}\left(f_{0}^{-1}(y)\right)$ for all $x, y \in X$.

Proof. According to Lemma 2 in [6], there is a finite open cover $\left\{W_{\beta}\right\}$ of $X$ such that, for each $\beta$ and for $i=0,1,2, \ldots, \operatorname{diam} W_{\beta}<\varepsilon / 2$ and $f_{i}$ maps every component of $f_{i}^{-1}\left(W_{\beta}\right)$ homeomorphically onto $W_{\beta}$. Let $\alpha$ be a Lebesgue number for $\left\{W_{\beta}\right\}$. It is easy to check that if $x, y \in X$ and $d\left(f_{i}(x), y\right)<\alpha$, then $B_{\alpha}^{\prime}(x) \cap f_{i}^{-1}(y)$ consists of a single point for each $i$ by the $\varepsilon$-local expansiveness of $f_{i}$. Here $B_{\alpha}^{\prime}(x)=\{z \in X$ : $d(x, z)<\alpha\}$. Since $f_{i} \rightarrow f_{0}$ uniformly, there is an $N_{1}$ such that $i \geqslant N_{1}$ implies $d\left(f_{i}(x), f_{0}(x)\right)<\alpha$ for all $x \in X$. Fix $x_{0} \in X$. Now suppose $i \geqslant N_{1}$ and $z \in f_{0}^{-1}\left(x_{0}\right)$. Then $d\left(f_{i}(z), x_{0}\right)<\alpha$. Thus, there is a unique $x^{\prime} \in X$ per $z$ such that $d\left(z, x^{\prime}\right)<\alpha$ $<\varepsilon / 2$ and $x^{\prime} \in f_{i}^{-1}\left(x_{0}\right)$. Hence, $\operatorname{card}\left(f_{i}^{-1}\left(x_{0}\right)\right) \geqslant \operatorname{card}\left(f_{0}^{-1}\left(x_{0}\right)\right)$. A similar argument shows that $\operatorname{card}\left(f_{i}^{-1}\left(x_{0}\right)\right) \leqslant \operatorname{card}\left(f_{0}^{-1}\left(x_{0}\right)\right)$. By Property 4.5 in [4], we get the conclusion.

Proof of TheOREM. Take $\eta$ with $0<\eta<\varepsilon / 6$. Let $\delta>0$ be as in the definition of the P.O.T.P. of $f_{0}$. Since $f_{i} \rightarrow f_{0}$ uniformly, there is an integer $N \geqslant N_{1}$ such that $i \geqslant N$ implies $d\left(f_{i}(x), f_{0}(x)\right)<\delta$ for all $x \in X$. Here $N_{1}$ is an integer given by Lemma 4. Then for $i \geqslant N$ there are unique continuous one-to-one (not necessarily onto) maps $h_{i}: X \rightarrow X$ with $h_{i} f_{i}=f_{0} h_{i}$ and $d\left(h_{i}(x), x\right)<\eta$ for all $x \in X$ (cf. [8, pp. 236-238]). By Lemma 4 we easily obtain that $f_{0}^{-1} h_{i}(X) \subset h_{i}(X)$ for $i \geqslant N$. Indeed, we may assume that there is an integer $k$ such that, for all $i \geqslant N$ and for all $x \in X$, $\operatorname{card}\left(f_{i}^{-1}(x)\right)=\operatorname{card}\left(f_{0}^{-1}(x)\right)=k$. Hence, for each $x^{\prime} \in h_{i}(X)$, there are exactly $k$-points $\left\{y_{1}, y_{2}, \ldots, y_{k}\right\}$ in $X$ such that $f_{i}\left(y_{m}\right)=h_{i}^{-1}\left(x^{\prime}\right)$ for $1 \leqslant m \leqslant k$ (since $h_{i}$ is one-to-one). Obviously, $f_{0}\left(h_{i}\left(y_{m}\right)\right)=h_{i}\left(f_{i}\left(y_{m}\right)\right)=x^{\prime}$ for $1 \leqslant m \leqslant k$. That is,

$$
f_{0}^{-1}\left(x^{\prime}\right)=\left\{h_{i}\left(y_{1}\right), h_{i}\left(y_{2}\right), \ldots, h_{i}\left(y_{k}\right)\right\} \subset h_{i}(X)
$$

(since $h_{i}$ is one-to-one and $\operatorname{card}\left(f_{0}^{-1}\left(x^{\prime}\right)\right)=k$ ). Hence, by Lemma 3 we have $h_{i}(X)=X$ for $i \geqslant N$. Let $a_{0}$ be a fixed point of $f_{0}^{n}$ and put $a_{i}=h_{i}^{-1}\left(a_{0}\right)$. Then $f_{i}^{n} h_{i}^{-1}=h_{i}^{-1} f_{0}^{n}$ implies $f_{i}^{n}\left(a_{i}\right)=a_{i}$, and $d\left(h_{i}(x), x\right)<\eta$ for all $x \in X$ implies $d\left(a_{i}, a_{0}\right)<\eta$ for all $i \geqslant N$ and for all $n \geqslant 1$.

ACKNOWLeDGement. The author is pleased to acknowledge many useful and pleasant conversations with Professor T. Kaneko. 


\section{REFERENCES}

1. N. Aoki, On homeomorphisms with the pseudo-orbit tracing property, Tokyo J. Math. 6 (1983), 330-334.

2. R. Bowen, Equilibrium states and the ergodic theory of Anosov diffeomorphisms, Lecture Notes in Math., vol. 470, Springer-Verlag, Berlin and New York, 1976.

3. E. M. Coven and W. L. Reddy, Positively expansive maps of compact manifolds, Global Theory of Dynamical Systems, Lecture Notes in Math., vol. 819, Springer-Verlag, Berlin and New York, 1980, pp. 96-110.

4. T. Hu and H. Rosen, Locally contractive and expansive mappings, Proc. Amer. Math. Soc. 86 (1982), 656-662.

5. W. L. Reddy, Expanding maps on compact metric spaces, Topology Appl. 13 (1982), 327-334.

6. H. Rosen, Fixed points of sequences of locally expansive maps, Proc. Amer. Math. Soc. 72 (1978), 387-390.

7. I. Rosenholtz, Local expansions, derivatives, and fixed points, Fund. Math. 91 (1976), 1-4.

8. P. Walters, On the pseudo-orbit tracing property and its relationship to stability, Lecture Notes in Math., vol. 668, Springer-Verlag, Berlin and New York, 1979, pp. 231-244.

Department of Mathematics, Faculty of Science, Niigata University, Niigata, Japan

Current address: Department of Mathematics, Tokyo Metropolitan University, Fukazawa, Setagaya-Ku, Tokyo, Japan 